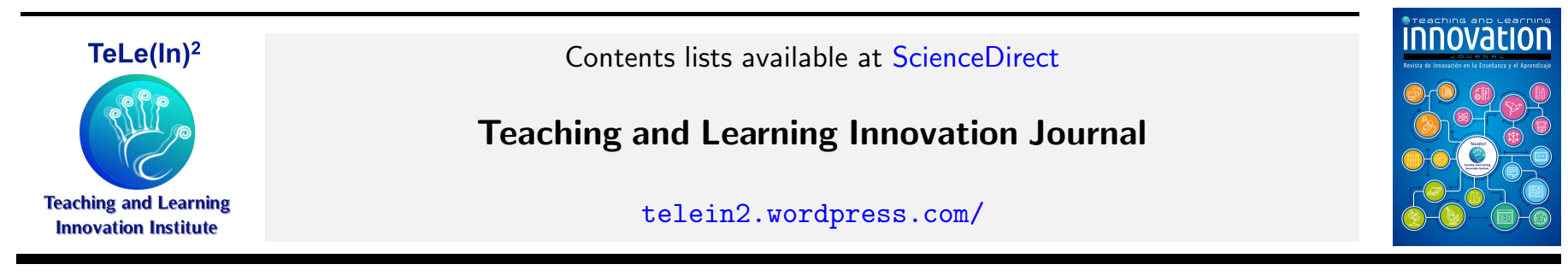

\title{
Aspectos críticos para aplicar con éxito el modelo flipped classroom a la enseñanza de la inmunología: resultados de 5 años de experiencias en la Universidad de Alcalá
}

\author{
Alfredo Prieto Martín ${ }^{\mathrm{a}}$, David Díaz Martín ${ }^{\mathrm{a}}$, Isabel Lara Aguilera ${ }^{\mathrm{a}}$, Jorge Monserrat Sanz ${ }^{\mathrm{a}}$, Raquel Oliva Martín ${ }^{\mathrm{a}}$, \\ José Barbarroja Escudero ${ }^{\mathrm{a}}$ \\ ${ }^{a}$ Departamento de Medicina y Especialidades Médicas, Universidad de Alcalá, Alcalá de Henares, Madrid, Spain
}

\begin{abstract}
Profesores de inmunología de la Universidad de Alcalá hemos implementado en los últimos cinco años el modelo de aula inversa en tres asignaturas del grado de Biología Sanitaria. El porcentaje de alumnos que estudiaron los materiales instructivos (documentos y vídeos) antes de que los temas fuesen tratados en clase ha aumentado desde valores minoritarios en los primeros años a porcentajes cercanos al $100 \%$ en los últimos años. La calificación media obtenida por los alumnos en las pruebas de evaluación del aprendizaje ha mejorado en las tres asignaturas en más de una desviación estándar con respecto a la que se obtenía con metodologías expositivas tradicionales antes de la implantación del modelo de aprendizaje inverso. Los porcentajes de alumnos que fracasan han disminuido y han aumentado los porcentajes de alumnos que alcanzan un nivel de maestría (calificación ¿8 en una escala de diez). Los alumnos reconocen desarrollar competencias con las actividades realizadas en los seminarios. El grado de satisfacción de los alumnos también ha aumentado. Concluimos que con el modelo inverso los alumnos universitarios dedican más trabajo no presencial al estudio de sus asignaturas y a consecuencia de ello sus calificaciones en la pruebas de evaluación del aprendizaje mejoran significativamente

Immunology teachers form Alcala's University implementes flipped classroom model in the last five years into three immunology courses in sanitary biology university degree. The percentage of students that studied the instructive materials (documents and videos) before class has increased from minority values in the first years to percentages close to $100 \%$ in the last years. The mean grade obtained by the classes in the learning assessment exams has increased in the three courses in more than one standard deviation with respect to the original mean obtained in each course with traditional expositive methods before the implementation of the flipped learning model. The percentages of failed students have decreased and those of students that attain mastery levels (grades i $80 \%$ of the maximum grade) have increased. The students recognize that the flipped model helps them to develop competencies in the activities developed in the seminars. The degree of satisfaction with the teaching has also increased We can conclude that university students thanks to the flipped model devote more time of work to the study of flipped courses and as a consequence of this increase in study they learn more and obtain increased grades in the learning assessment exams.
\end{abstract}

Keywords: aula inversa, aprendizaje inverso, gamificación, inmunología

Flipped classroom, flipped learning, gamification, Immunology

\section{Introducción}

Dentro del modelo del aprendizaje inverso a nivel universitario se han usado desde los años 90 diversas metodologías de fomento del estudio previo a las clases que incluyen el Just in time teaching (Novak 1999), el Team based

Email addresses: alfredo.prieto@uah.es (Alfredo Prieto Martín), david.diaz@uah.es (David Díaz Martín), Jorge.monserrat@uah.es (Jorge Monserrat Sanz), Jose.barbarroja@uah.es (José Barbarroja Escudero)
Learning (Michaelsen 2002) y el Peer instruction (Mazur 1997). La metodología de blended learning (combinación mixta de aprendizaje presencial con aprendizaje que usa medios electrónicos) just in time Teaching (enseñanza justo a tiempo) fue desarrollada por Gregor Novak en los 90 y se basa en la comunicación bidireccional entre el profesor y sus alumnos por medios electrónicos antes de la interacción presencial en el aula (Novak, 1999).

Los profesores del área de medicina e inmunología del 
Departamento de Medicina y Especialidades Médicas de la Universidad de Alcalá llevamos años implementando con gran éxito una variante del JITT que denominamos flipped classroom with just in time teaching (FC/JITT) (Prieto y cols. 2014). En este método se usan cuestionarios on line en los que preguntamos a nuestros alumnos sobre los materiales instructivos que les hemos enviado con antelación. Este método nos permite también obtener conclusiones sobre las reacciones de nuestros alumnos a los materiales instructivos proporcionados y usar esta información proveniente de los alumnos para a continuación proporcionarles retroinformación o feedback que les ayude a superar sus dificultades para comprender y aprender.

Esta comunicación bidireccional entre profesores y alumnos permite crear un ambiente de comunidad de aprendizaje en el que el profesor tiene conocimiento de las dificultades e intereses de sus alumnos (Novak, 1999). Así los profesores entendemos qué es lo que nuestros alumnos no logran comprender en los materiales instructivos que les facilitamos (Prieto 2014). Este sistema primero nos permitió primero detectar los problemas de comprensión más prevalentes entre nuestros alumnos en los temas que les impartíamos en nuestras asignaturas. Esta información nos sirvió para preparar nuevos materiales instructivos, actividades y ejemplos que ayudasen a que nuestros alumnos comprendiesen mejor esos conceptos.

En este artículo mostramos que el uso de esta metodología produce un impacto muy positivo en cuanto al trabajo de estudio previo de los alumnos, en cuanto a la mejora de las calificaciones y en cuanto a la opinión de los alumnos sobre la docencia que reciben. Finalmente, discutimos las razones que pueden subyacer a esta mejora en los resultados del aprendizaje y nos enfocamos en como otros profesores universitarios pueden analizar las respuestas de nuestros alumnos y usar las conclusiones de dicho análisis para adaptar nuestras clases a las necesidades de nuestros alumnos.

El análisis de respuestas de los alumnos a los cuestionarios de comprobación del estudio previo a los que los alumnos responden en el FC/JITT nos sirve para poder responder a preguntas muy relevantes acerca de las reacciones de nuestros alumnos tras interaccionar con los materiales instructivos. Preguntas de este tipo incluyen: ¿Qué es lo que más interesa a mis alumnos?, ¿Qué es lo que no necesitan que les explique?, ¿Qué es lo que es urgente explicar?, ¿Qué alumnos se han preparado para la clase?, ¿Cuánto tiempo trabajan?, ¿A qué profundidad intentan comprender?, ¿Cómo de bien están haciendo el trabajo de preparación?

\section{Contexto}

Los nuevos grados del Espacio Europeo de Educación Superior, orientados hacia el desarrollo de competencias y basados en un tipo de crédito ECTS en el que debe predominar el trabajo no presencial del alumno, demandan un profundo cambio en la metodología de enseñanza universitaria. En este contexto de la enseñanza universitaria moderna es necesario implementar metodologías que fomenten el estudio de los alumnos y permitan usar parte del tiempo de clase en actividades. La nueva metodología debe en primer lugar estimular a los alumnos a estudiar más fuera de clase. En segundo lugar, debe permitirnos utilizar el tiempo de clase no para la exposición de la información a ser aprendida, sino para que los alumnos sean protagonistas activos que apliquen conocimientos que han recibido por medios electrónicos y han asimilado por medio de estudio autónomo. Las nuevas metodologías deben adaptarse a las necesidades de los alumnos individuales para así poder aportar a cada uno lo que sea necesario para optimizar su experiencia de aprendizaje. Esto requiere un análisis de las reacciones de los alumnos a los materiales instructivos que les proporcionamos para que inicien su aprendizaje.

En el contexto del modelo de aprendizaje inverso, el análisis del aprendizaje sirve para obtener evidencias de cómo está aprendiendo un alumno a partir de fuentes diversas: su interacción con los contenidos, sus respuestas a cuestionarios de comprobación del estudio, sus respuestas a ejercicios y actividades de evaluación formativa presencial con Sistemas de Respuesta Personal en el aula o mediante pruebas on line (Bruff, 2009). El análisis del aprendizaje pretende relacionar los logros de los alumnos con los patrones de comportamiento y actividad de cada alumno, predecir si los estudiantes necesitarán apoyo y atención diferenciada o personalizada e identificar precozmente a los alumnos que necesitan la intervención urgente del profesor (Amo, 2014).

Los objetivos de este trabajo son difundir unas experiencias que han dado muy buenos resultados en asignaturas universitarias de distintos cursos de grado universitario e ilustrar los aspectos críticos para que otros profesores puedan empezar a incorporar a su enseñanza estas metodologías de aprendizaje inverso. Por tanto, este artículo se dirige a profesores universitarios que desean incorporar el aprendizaje inverso a sus asignaturas y para ello deben ser capaces de fomentar el estudio preparatorio entre sus alumnos así como ser capaces de analizar sus respuestas y usarlas para generar actividades de clase.

\section{Descripción}

Factores críticos para el éxito en la implementación del modelo inverso o flipped

Fomentar el compromiso de los alumnos para realizar el estudio previo de manera regular es crítico para lograr que los alumnos aprendan más. Para conseguir ese compromiso de los alumnos al inicio de la asignatura los profesores debemos aportarles motivos para que cambien sus hábitos y se comprometan a estudiar los temas antes de que sean tratados en clase. Tras este estudio de preparación deberán responder a unos cuestionarios que servirán para 
que los profesores comprueben su trabajo, lo puedan premiar, y estén informados de sus intereses y dificultades con respecto al tema tratado.

A este logro contribuye tanto un marketing al inicio de la asignatura como una labor día a día que incluye responder a sus preguntas urgentes (flipped Learning forte), replantear las clases en función de los intereses y necesidades manifestados en sus respuestas (Just in time Teaching), reconocer públicamente a los que hacen bien este trabajo (Hall of fame).

El análisis de respuestas de nuestros alumnos también nos permite identificar a aquellos alumnos que se implican menos en las actividades de fomento del estudio previo y recriminar en privado a los que no realizan el estudio previo (mails privados a los que no trabajan). El análisis también permite identificar a aquellos alumnos que realizan el estudio con un enfoque más superficial (Biggs 1999). A estos alumnos les podemos enviar un e-mail en el que les comunicamos que "tus respuestas me demuestran que todavía no te has esforzado lo suficiente para entender el tema. Vuelve a estúdiatelo mejor y responde de nuevo al cuestionario".

También es crítico implementar un método eficaz de comprobación del trabajo previo. Nosotros empleamos un cuestionario on line por ejemplo en Google forms nos aportara información sobre: qué alumnos han hecho el estudio previo, qué les interesa más, qué es lo que han comprendido bien y no necesitan que les expliquen, qué partes del tema permanecen oscuras después de haber intentado comprenderlas, cuáles son las dudas urgentes que necesitan que les expliquen con más urgencia y cuánto tiempo han tenido que estudiar para prepararse. Al cuestionario reflexivo universal que nos vale para cualquier tema, le podemos añadir preguntas específicas sobre la información contenida en el tema. Estas preguntas sobre recuerdo o comprensión de información específica pueden ser de elección entre múltiples respuestas. También es importante desarrollar un sistema eficaz para el análisis de respuestas de los alumnos. A continuación, ofrecemos procedimientos de análisis de las respuestas a las distintas preguntas del cuestionario para llegar a conclusiones sobre los intereses y necesidades de nuestros alumnos. También ofrecemos ejemplos de casos reales del uso de estos procedimientos para detectar lo que debe ser tratado de manera prioritaria en clase y sobre lo que puede omitirse con o tratarse de una manera menos profunda.

\section{Métodos de proceso y análisis de la información contenida en las respuestas a los cuestionarios}

El proceso de análisis de respuestas en Excel requiere la realización de una serie de operaciones de forma ordenada:

1. Separado de columnas para aumentar su legibilidad. Se corta la columna de respuestas (por ejemplo ¿Qué es lo que te parece más difícil de entender?) y se pega en una nueva hoja. La anchura de la columna se aumenta lo que haga falta para aumentar la legibilidad de las respuestas y finalmente se autoajusta la altura de celda.

2. Categorización de respuestas de los alumnos por etiquetado en categorías discretas

3. Ordenación alfabética de la columna de etiquetas

4. Cuantificación de cuantos alumnos no entienden cada tópico.

5. Elaboración de rankings de conceptos más difíciles de entender y representación visual de los mismos.

Con esta información podemos tomar decisiones sobre qué es necesario priorizar en clase, qué actividades son más necesarias y qué explicaciones son prescindibles.

Métodos de flipped learning 2.0 para convertir las dudas de nuestros alumnos en fuentes de feedback y actividades para clase: Flipped Learning Forte $y$ flip in colours

Nuestra forma de utilizar las respuestas de nuestros alumnos para proporcionarles feedback y crear actividades para clase ha ido evolucionando. En los dos últimos cursos hemos utilizado metodologías mejoradas para proporcionar más y mejor feedback a nuestros alumnos sobre sus dudas urgentes (flipped learning forte y flip in colours).

En el flipped learning forte el profesor contesta en una lista de distribución a las dudas urgentes de sus alumnos. Todos los alumnos reciben la respuesta del profesor a sus propias dudas urgentes y a las de todos sus compañeros. En el flip in colours el profesor clasifica las dudas urgentes de sus alumnos y decide cual es el mejor uso de cada duda. Unas deberá responderlas el profesor en clase, otras podrán resolverlas los alumnos con nivel de comprensión más avanzada y explicárselas a sus compañeros, otras servirán de inspiración para dar pie a proyectos de indagación, debates en clase, e incluso preguntas de examen. Así en la metodología flip in colours el profesor clasifica las dudas en función de su posible uso y las usa en clase para generar preguntas para que sean respondidas o por los propios alumnos o debatidas en clase. De modo complementario el profesor puede escoger algunas dudas especialmente interesantes y responderlas o discutirlas con los alumnos en clase.

Primero ideamos y desarrollamos el método aprendizaje inverso fuerte (flipped learning forte) que consiste en responder a la duda urgente que cada alumno ha planteado en su cuestionario, pero en lugar de enviar a cada alumno solamente la respuesta a su duda particular, las respuestas a las dudas de todos los alumnos que han respondido al cuestionario se envían a una lista de distribución de e-mail que incluye a todos los alumnos de la clase incluso a los que no han realizado el estudio previo y no han respondido al cuestionario. De esta manera hasta aquellos alumnos que no han realizado el estudio previo reciben el feedback de sus profesores sobre las dudas de los compañeros que si han realizado el estudio previo.

En el último curso hemos ideado e implementado la metodología Flip in colours: Black, Yellow background, 
Green, Red, Blue, Orange, Pink en la que clasificamos las dudas urgentes de nuestros alumnos en cinco categorías que marcamos con distintos colores en las matrices de datos que contienen las respuestas de nuestros alumnos tras el estudio previo de los temas.

Se marcan en negro y en estilo negrita aquellas dudas que requieren ser respondidas por el profesor.

En negro con fondo amarillo aquellas dudas que requieren una indagación por el profesor antes de ser respondidas.

En verde las que pueden ser respondidas por los propios alumnos con un nivel de comprensión más avanzado.

En rojo las que implican un error conceptual que deber ser señalado y corregido.

En azul las que pueden inspirar buenas preguntas para las pruebas de evaluación.

En naranja aquellas preguntas que pueden ser buenas para iniciar discusiones y debates en el aula.

Finalmente, en rosa aquellas que pueden servir para iniciar actividades de indagación o aprendizaje por medio de problemas y proyectos.

En función del tipo de duda de que se trate se usan de distinta manera en el aula por ejemplo se puede pedir a los alumnos que expliquen las verdes o que intenten averiguar cuál es el error conceptual que subyace a una pregunta roja o que preparen una respuesta para la azul, que debatan sobre la naranja o que indaguen o realicen $\mathrm{ABP}$ sobre las rosas.

\section{Resultados}

Para medir el impacto del cambio metodológico cuantificamos en cada clase el porcentaje de alumnos que realizaban el estudio previo de más de la mitad de los temas y la calificación media obtenida en las pruebas de evaluación del aprendizaje. A continuación se muestra la evolución de estas variables en promociones sucesivas en las que fuimos implementando mejoras en nuestra metodología.

\section{A. Estudio previo}

En el primer año de implementación del FC/JITT en las tres asignaturas Inmunología clínica de cuarto curso (izquierda), inmunología de tercer curso centro y Métodos en biología de primer curso, los porcentajes de alumnos que estudiaron al menos la mitad de los temas fueron inferiores al $50 \%$. En las dos asignaturas (izquierda y centro) en las que al segundo año hicimos marketing de la metodología al inicio de la asignatura los porcentajes subieron, pero quedaron por debajo del $70 \%$. Sin embargo, cuando al principio de las asignaturas se anunciaron bonificaciones en la calificación a los alumnos que hiciesen el estudio previo conseguimos superar el umbral del $70 \%$ en las tres asignaturas.

Calificaciones medias en las pruebas de evaluación del aprendizaje
En las tres asignaturas del grado en biología sanitaria en las que hemos implementado el modelo flipped las notas medias obtenidas en las pruebas de evaluación mejoran con respecto a las que se obtenían con metodología tradicional.

En las tres asignaturas cuando el porcentaje de alumnos que realizan el estudio previo supera el 70 por ciento se observan aumentos de la nota media cercanos o superiores a una desviación estándar con respecto a las que se obtenían antes de la implementación del modelo inverso. Para hacerse una idea del tamaño de efecto de una subida de una desviación estándar ayuda el considerar que con el modelo flipped el alumno en el percentil 50 alcanza niveles de aprendizaje que igualan a los que obtenía el alumno en el percentil 84 cuando utilizábamos el método expositivo tradicional.

Impacto sobre la valoración de los alumnos

El empleo del flipped learning forte nos ha permitido obtener mejoras, tanto en las calificaciones que los alumnos obtienen en las pruebas de evaluación del aprendizaje, como en las valoraciones obtenidas por los profesores en las encuestas de opinión sobre la docencia de los alumnos. Así la media de valoración de la docencia por los alumnos en la asignatura de inmunología clínica subió desde un 6 en el curso $13 / 14$ a un 7,6 2 en el curso 14/15, a un 8,6 en el curso $15 / 16$ y a un 9,1 en el 16/17 (Figura 3).

En el último curso hemos llegado a una valoración record por encima de los 9 puntos sobre 10 (Figura 4).

\section{Conclusiones}

El impacto positivo del modelo inverso sobre la mejora del aprendizaje de los alumnos universitarios ha sido demostrado por numerosos autores (Mazur Knight \& Woods, 2005; Huston, 2009). Nuestra propia experiencia es que las calificaciones medias en las pruebas de evaluación del aprendizaje suelen subir en una magnitud similar a una desviación estándar de la distribución de notas original. Esto supone que el alumno mediana (percentil 50) con el modelo inverso adquiere niveles de aprendizaje similares a los que obtenía con metodología tradicional el alumno del percentil 84. A la vista de las mejoras en aprendizaje obtenidas con esta combinación de métodos de aula inversa (mejora de calificaciones medias en pruebas de evaluación del aprendizaje, disminución de tasa de fracaso, aumento del porcentaje de alumnos que alcanzan nivel de maestría) cabe preguntarse sobre cuáles son las razones que explican la eficacia de este modelo de aprendizaje.

Con el modelo de aprendizaje inverso logramos que un mayor porcentaje de alumnos estudien para las clases y se impliquen en las clases. También logramos que más alumnos aprueben y que los que lo consiguen hagan trabajos de mejor calidad y obtengan mejores calificaciones. El proceso de preparación y de trabajo en clase contribuye a detectar y esclarecer los conceptos erróneos, ayuda a profundizar la comprensión y a dominar las abstracciones y la capacidad de transferencia a nuevas situaciones. El modelo inverso 


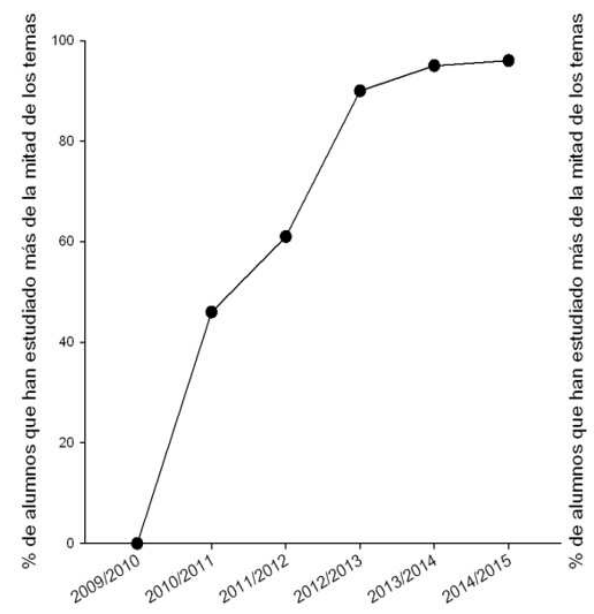

Año académico

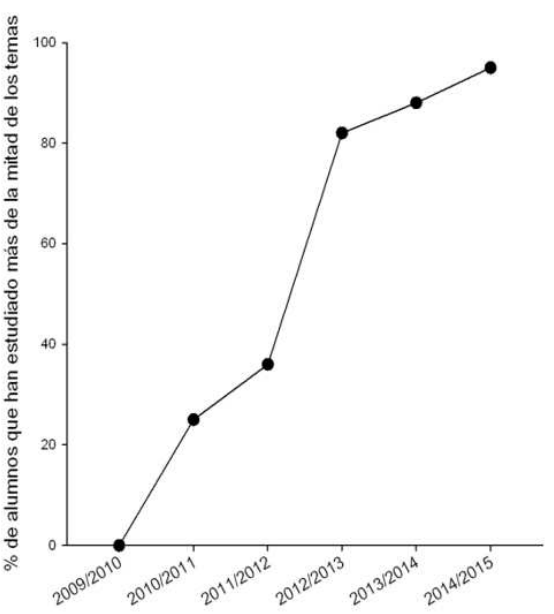

Año académico

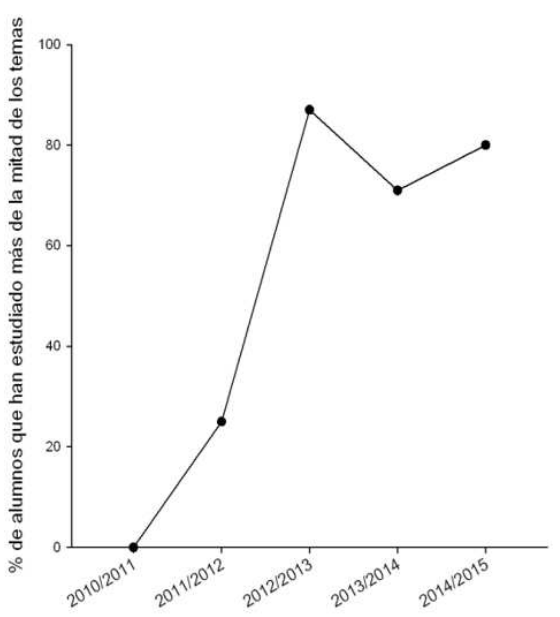

Año académico

Figura 1: Porcentaje de alumnos que realizó el estudio previo y realizo las tareas de preparación para las clases en las asignaturas de inmunología clínica de $4^{\circ}$ curso, inmunología de $3^{\circ}$ curso y métodos en biología (parte de inmunología) de $1^{\circ}$. Obsérvese que a partir del curso 2012/13 cuando se implementó el sistema de bonificación por el estudio previo los porcentajes de alumnos que realizaron el estudio previo fueron superiores al $70 \%$ en las tres asignaturas.

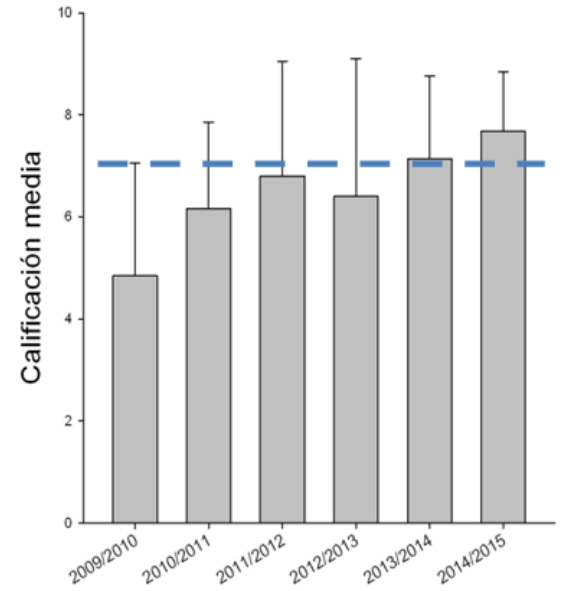

Año académico

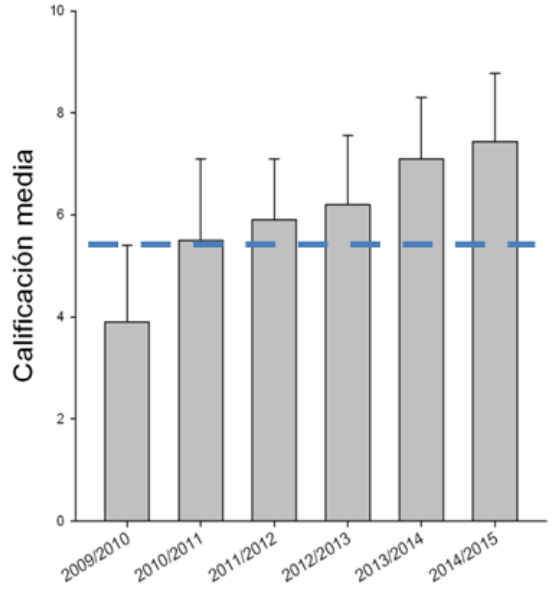

Año académico

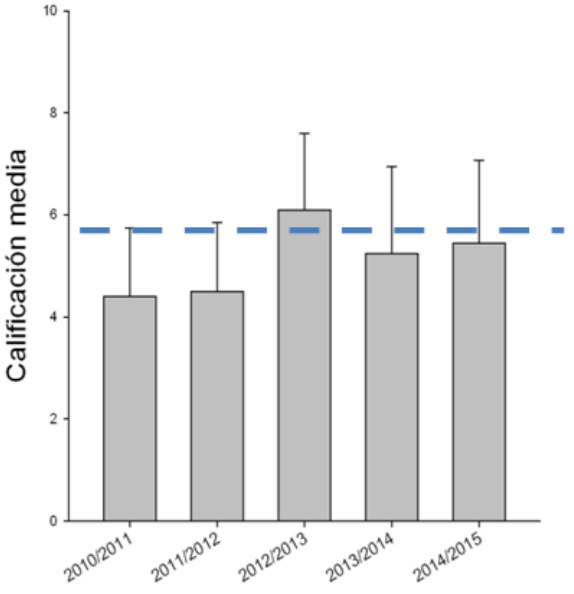

Año académico

Figura 2: Calificaciones medias (+desviación estándar) en las asignaturas de inmunología clínica de cuarto curso, inmunología de tercer curso y métodos en biología (parte de inmunología) de primer curso. Las líneas discontinuas de cada gráfica representan el nivel de una desviación estándar por encima de la media de la calificación obtenida con la metodología tradicional. 


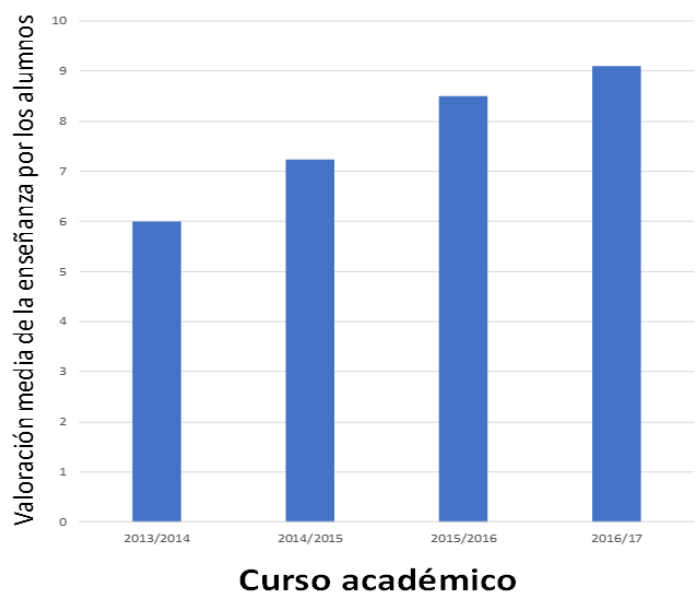

Figura 3: Con las metodologías flipped learning forte y flip in colors las valoraciones de los alumnos en las encuestas oficiales de opinión de los alumnos sobre la docencia mejoran espectacularmente (cursos 2014/15, 2015/16 y 2016/17).

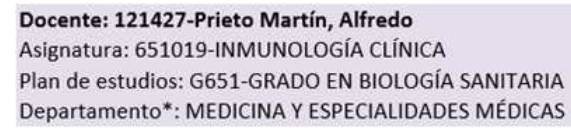

\begin{tabular}{|c|c|c|c|c|c|c|c|c|c|}
\hline \multirow{3}{*}{ Pregunta } & \multirow{2}{*}{\multicolumn{3}{|c|}{ Docente }} & \multirow{2}{*}{\multicolumn{3}{|c|}{ Departamento* }} & \multirow{2}{*}{\multicolumn{3}{|c|}{ UAH* }} \\
\hline & & & & & & & & & \\
\hline & Media & DT & n & Media & DT & n & Media & DT & $\mathbf{n}$ \\
\hline P1: La claridad de sus explicaciones & 7.7 & 1.3 & 15 & 6.7 & 3.2 & 396 & 7.3 & 2.7 & 17,144 \\
\hline P2: Su corrección y respeto hacia los/las estudiantes & 9.8 & 0.4 & 15 & 7.8 & 2.8 & 390 & 8.0 & 2.6 & 16.989 \\
\hline P4: Su capacidad para fomentar la participación de los/las estudiantes en clase & 9.5 & 0.5 & 15 & 6.5 & 3.2 & 386 & 7.1 & 2.9 & 17.042 \\
\hline P4: El ajuste de los contenidos impartidos con lo previsto en la Guía Docente & 8.1 & 1.6 & 15 & 7.0 & 3.2 & 378 & 8.0 & 2.5 & 16.409 \\
\hline P5: Su disponibilidad en las tutorías & 9.6 & 0.7 & 12 & 6.9 & 3.3 & 257 & 8.0 & 2.6 & 12.578 \\
\hline P6: La coherencia de los métodos de evaluación empleados con el tipo de asignatura & 9.6 & 0.5 & 15 & 6.9 & 3.3 & 361 & 7.6 & 2.8 & 16,450 \\
\hline P7: El ajuste de la evaluación con lo previsto en la Guía Docente & 9.4 & 0.6 & 15 & 7.2 & 3.2 & 349 & 8.0 & 2.6 & 15.666 \\
\hline TOTAL & 9.1 & 1.2 & 102 & 7.0 & 3.2 & 2,517 & 7.69 & 2.7 & 112,278 \\
\hline
\end{tabular}

Estadisticos: Media="media aritmética", DT="desviación típica", n="número de respuestas".

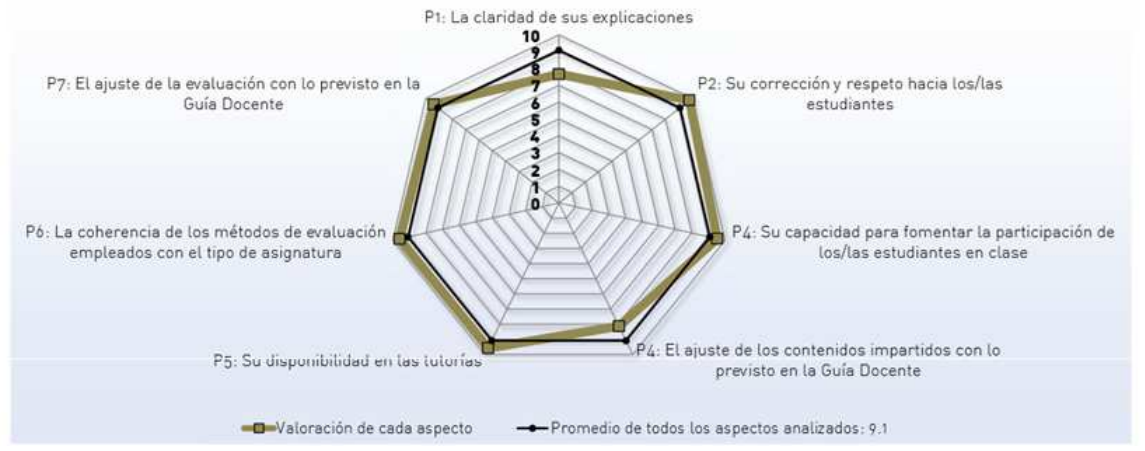

Figura 4: Con las metodologías propuestas, flipped learning forte y flip in colors las valoraciones de los alumnos en las encuestas oficiales de opinión de los alumnos sobre la docencia son espectacularmente buenas. Valoración correspondiente al curso 2016/17. 
también contribuye a mejorar las actitudes en clase y la predisposición a la participación. Estos mismos efectos positivos han sido observados por otros autores al incorporar aprendizaje activo a sus clases (Knight \& Woods, 2005; Huston, 2009).

Finalmente, aunque en ocasiones hemos observado que inicialmente la implementación de un modelo flipped puede causar resistencia y reacciones adversas en algunos estudiantes que prefieren seguir usando el siempre más cómodo método tradicional al final estas reacciones se superan especialmente a largo plazo y sobre todo cuando los profesores somos capaces de demostrar a nuestros alumnos que trabajamos con sus respuestas y cambiamos nuestros planes de clase en base a ellas dándoles feedback y modificando materiales y actividades para clase.

\section{Referencias}

[1] Amo D Santiago R (2017) Learning Analytics La narración del aprendizaje a través de los datos. Barcelona Universitat oberta de Catalunya

[2] Biggs J. (1999) What the student does: teaching for enhanced learning. Higher Education Research and Development 18:57-75

[3] Bruff D (2009) Teaching with Classroom Response Systems: Creating Active Learning Environments. Jossey-Bass San Francisco

[4] Knight J y Wood WB (2005) Teaching more by lecturing less Cell Biol Educ. Winter; 4(4): 298-310

[5] Huston T (2012) Teaching what you don't know. Cambridge. Harvard University Press

[6] Mazur E. (1997) Peer instruction: A User's Manual. Prentice Hall Series in Educational Innovation Englewood Cliffs, NJ: Prentice Hall.

[7] Michaelsen, L.K., Knight, A.B., y Fink L.D. (2002) TeamBased Learning: A Transformative Use of Small Groups in College. Westport: Praeger Publishers.

[8] Novak G, Gavrin A, Christian W, Patterson E. (1999) Just-In-Time Teaching: Blending Active Learning with Web Technology. Upper Saddle River,NJ: Prentice-Hall.

[9] Prieto A., Díaz D., Monserrat J., Reyes E. (2014a). Experiencias de aplicación de estrategias de gamificación a entornos de aprendizaje universitario. Revisión, 7 (2): 76-92.

[10] Prieto A. (2014) ¿Trabajan en su aprendizaje nuestros alumnos universitarios lo que deberían? Sobre cerditos y créditos ECTS: el cuento de los dos cerditos del espacio europeo. Profesor 3.0 URL:http://profesor3punto0.blogspot.com.es/2013/05/sobrecerditos-y-creditos-ects-el.html

[11] Prieto A (2017) Flipped Learning: Aplicar el modelo de Aprendizaje Inverso Madrid Editorial Narcea

[12] Sams A, Bergmann J. (2014) Flipped learning gateway to student engagement. International Society for Technology in Education.

[13] Sams A, Bergmann J. (2012) Flip your Classroom: Reach every student in every class every day. International Society for Technology in Education. 